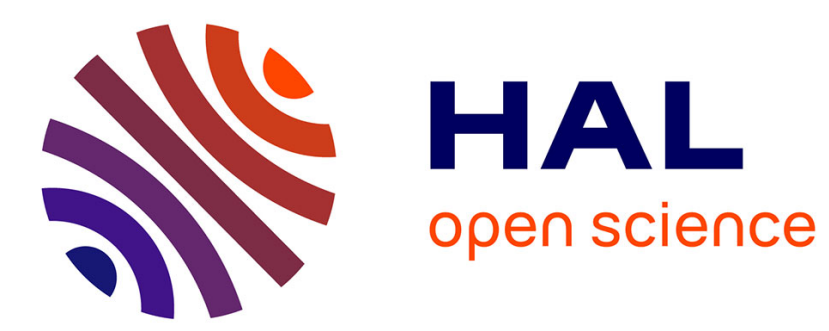

\title{
GEMINATE AND NON-GEMINATE RECOMBINATION IN AMORPHOUS SEMICONDUCTORS
}

\author{
J. Mort
}

\section{> To cite this version:}

J. Mort. GEMINATE AND NON-GEMINATE RECOMBINATION IN AMORPHOUS SEMICONDUCTORS. Journal de Physique Colloques, 1981, 42 (C4), pp.C4-433-C4-441. 10.1051/jphyscol:1981491 . jpa-00220948

\section{HAL Id: jpa-00220948 https://hal.science/jpa-00220948}

Submitted on 1 Jan 1981

HAL is a multi-disciplinary open access archive for the deposit and dissemination of scientific research documents, whether they are published or not. The documents may come from teaching and research institutions in France or abroad, or from public or private research centers.
L'archive ouverte pluridisciplinaire HAL, est destinée au dépôt et à la diffusion de documents scientifiques de niveau recherche, publiés ou non, émanant des établissements d'enseignement et de recherche français ou étrangers, des laboratoires publics ou privés. 
J. Mort

Xerox Webster Research Center, 800 Phillips Road - 114, Webster, N.Y. 14580, $U . S . A$.

Abstract.- One of the characteristic features of amorphous semiconductors is the low carrier mobilities which result from small carrier mean-free-paths due to disorder. When the mean-free-path becomes comparable to the Coulomb radius of an electronhole pair, both photogeneration and recombination processes can be affected. The photogeneration process can be controlled by geminate or initial pair recombination which results in a field-dependent photogeneration efficiency. The non-geminate recombination of previously created free carriers can become diffusion-controlled. This paper reviews the evidence for the occurrence of such processes in amorphous semiconductors including recent studies in a-Si:H and a- $\mathrm{As}_{2} \mathrm{Se}_{3}$.

Introduction.- Intense technological interest exists in hydrogenated amorphous silicon, a-Si:H, for applications as photovoltaic cells [1,2], thin film electronic devices [3,4] and electrophotographic photoreceptors [5,6]. Optimization and refinement of the properties of the material for such applications requires some fundamental understanding of their photoelectronic properties. The considerable progress which has been made in this respect is the subject of a recent excellent review[7]. The purpose of this paper is to discuss the photogeneration and recombination processes in a-Si: $\mathrm{H}$ in relation and comparison to other amorphous materials such as amorphous selenium a-Se, amorphous arsenic triselenide, $\mathrm{a}-\mathrm{As}_{2} \mathrm{Se}_{3}$ and amorphous molecular solids. The unifying characteristic of these materials is that they possess low carrier mobilities. In view of this fact the photogeneration and recombination of carriers may be fundamentally different from similar and more familiar processes in crystalline semiconductors [8]. An understanding of such differences and their manifestation, in particular amorphous solids, is obviously an important precursor to any device improvement.

Low carrier mobilities are a consequence of small carrier mean-free-paths associated with strong carrier scattering due to the disorder. The best estimates indicate microscopic mobilities in amorphous solids are limited to $<10 \mathrm{~cm}^{2} / \mathrm{Vsec}[9,10]$ while shallow trapping leads to still lower drift mobilities [9]. Such small carrier mean-free-paths can influence both the initial photogeneration step and the subsequent recombination of previously created carriers. If the photoexcited electron-hole pair thermalize at a distance $r_{0}$, (the thermalization radius) which is comparable to the Coulomb radius, $r_{c}$, $\left(r_{c}\right.$ is the distance at which the Coulomb energy is $\sim \mathrm{kT}$ ) then recombination can occur even before free carrier creation occurs [see Figure 1(a)]. This is the so-called geminate or initial recombination. Its primary consequence is to result in a field, temperature and, in the case of a-Se, a wavelength dependent photogeneration efficiency. The relative value of $r_{0}$ with respect to $r_{c}$ and the quantum efficiency of production of initially thermalized pairs $\Phi_{0}$ determine the quantum efficiency of photogeneration in zero field $\Phi(0)$. Such a process can be analytically described by a theory due to Onsager [11]. The characteristic features of a geminate process are a finite quantum efficiency at zero field with a value determined by $\Phi_{0}, r_{0}$ and $r_{c}$. Up to fields of $\sim 10^{4} \mathrm{~V} / \mathrm{cm}$ this quantum efficiency is essentially constant beyond which it rises with increasing field to ultimately saturate at the value $\Phi_{0}$. Thus for a higher value of $\Phi(0)$ (for given values of $\Phi_{0}$ and $r_{c}$ this is determined by $r_{0}$ ) the weaker the field dependence in the field-dependent regime. 
In Figure 1(b) the case of the recombination of previously free electrons and holes is considered. It is assumed that a density $\mathrm{N}_{\mathrm{r}}$ of recombination centers with a capture cross-section $\sigma$ exist and that the density of photogenerated carriers exceeds the dark equilibrium carrier density. In a large mobility solid such as a crystal, if the volume swept out by the carrier moving with a thermal velocity $v_{T}$ in a scattering time $\tau_{\mathrm{s}}$ encompasses a recombination center [ie $v_{\mathrm{T}} \tau_{\mathrm{s}}$ $\left.>\left(N_{r}\right)^{-1 / 3}\right]$, recombination will occur and the recombination coefficient $b=v_{T} \sigma$ [12]. If, however, for a low mobility solid where $v_{T} \tau_{s} \ll r_{c}$, then a carrier which moves within the mutual Coulomb radius of an oppositely charged carrier will suffer collisions within the Coulomb well and will diffuse to ultimate recombination [13]. Such nongeminate recombination for a low mobility solid will then be diffusion-controlled. This type of process is similar to the recombination of ions in a dense gas first treated by Langevin [14].

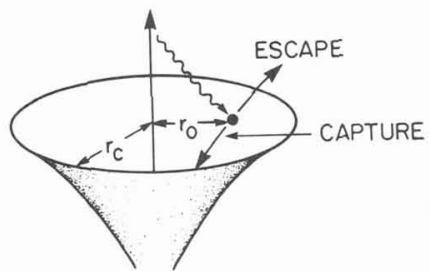

(a)
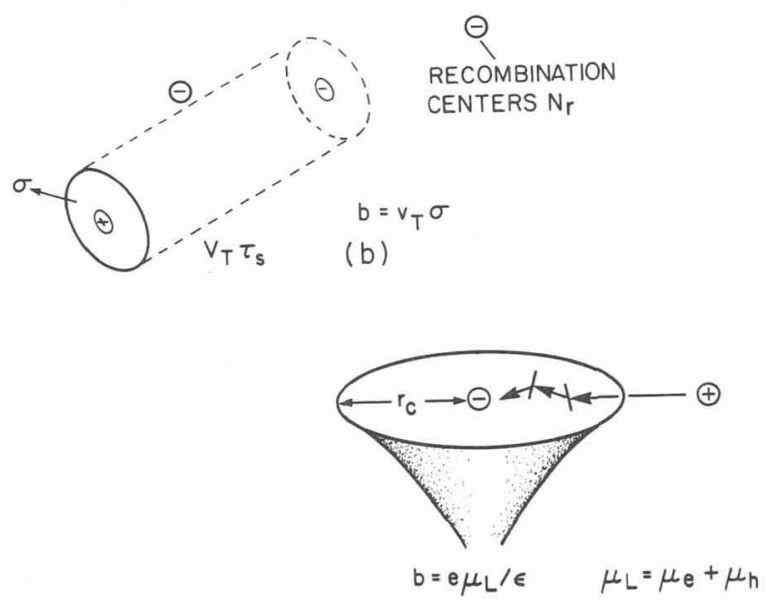

(c)

Fig. 1. Schematic representations of (a) geminate recombination, (b) non-geminate recombination in high mobility solids and (c) non-geminate recombination in low mobility solids.

In this case the recombination coefficient $b=e \mu_{L / \varepsilon}$ where $\mu_{\mathrm{L}}$ is the Langevin mobility and equals the sum of the electron and hole drift mobilities and $\varepsilon$ is the dielectric constant. An identifying feature of such a process therefore is a recombination lifetime which has a temperature dependence consistent with those of the drift mobilities.

\section{Geminate Recombination}

One of the best examples of geminate recombination-controlled photogeneration is the case of a-Se. Figure 2 shows the photoinjection efficiencies for holes in a-Se as a function of applied electric field for eight different exciting wavelengths and two sample thicknesses. The data shown were obtained by Pai and Enck [15] using the xerographic discharge technique. For fields larger than about $10^{3} \mathrm{~V} / \mathrm{cm}$ the samples of two different thicknesses have identical efficiencies. This demonstrates that bulk trapping is negligible in this field range and the field dependence is associated with recombination and/or trapping in the excitation region. By itself the data only establish that there is a loss process operative in the excitation region. The solid lines are theoretical calculations of the Onsager theory assuming $\Phi_{0}=1$, a dielectric constant of 6.3 and different values of the thermalization distance $r_{0}$. The extremely good fit in the high field regimes implies that the thermalization distance is wavelength dependent, a feature explained by Knights and Davies [16]. This data disagrees strongly with the Onsager model in 
the critical low field regime. However, additional experiments including twophoton photogeneration and delayedcollection field experiments [15] established that the zero field quantum efficiency was in accord with predictions and the low field deviation of the photoinjection efficiency measured xerographically is assoeiated with surface recombination or trapping. It is interesting that such a microscopic process which results in a wavelength dependent $r_{0}$ manifests itself in the macroscopic obser that a-Se although panchromatic in absorption is not so in photoconductivity.

Recent xerographic discharge studies on thick a-Si: $\mathrm{H}$ samples $<30 \mu \mathrm{m}$ shows many similarities to a-Se. Figure 3(a) shows a plot of the quantum efficiency as a function of electric field. The open circles refer to xerographic discharge measurements while the solid lines are Onsager curves calculated with $\Phi_{0}=I$ and $\varepsilon=11.5$. As in the case of a-Se the data, while consistent with the Onsager predictions at high fields, deviate markedly at low fields. The closed symbols represent the measurements using the delayed collection field technique on the same sample. For details of the experiment the reader is referred to published work

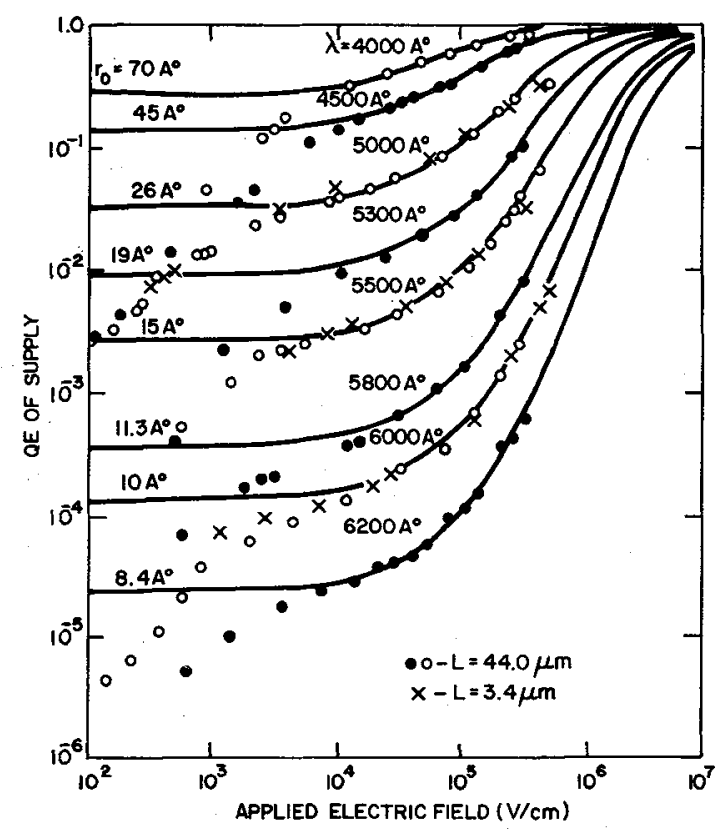

Fig. 2. Experimental data of the quantum efficiency of photoinjection of holes as a function of electric field for a-Se films of two different thicknesses. Included are the theoretical Onsager dissociation efficiencies (solid lines) for $\Phi_{0}=1$ and for different thermalization distances $r_{0}$ (Ref. 15).

[17] but suffice to say the experiment allows the creation of carriers with variable field, including zero, which, followed within 50 nanoseconds by a high sweep-out field, allows collection of all the photogenerated carriers without loss. This latter fact is checked by studying the loss of carriers within the excitation region as the delay before applying the collection field is increased. The data shown are for a-Si: $\mathrm{H}$ samples made with, (a) an rf power of 18 watts with a silane concentration of $5 \%$ in $\mathrm{He}_{\text {, }}$ (b) samples made with rf power of 2 watts with $100 \%$ silane. Zerofield efficiencies are found ranging from $0.4-0.55$ which are consistent with thermalization lengths ranging from $40-80 \AA$. Unlike a-Se the photoconductivity in a-Si:H coincides with its absorption edge which means that $r_{0}$ has at most a very weak wavelength dependence. An important check on the origin of the carrier loss within the excitation region is that a geminate recombination process described by the Onsager formalism has a very precise prediction. Namely, once $\Phi_{0}, r_{c}$ and $r_{0}$ are fixed the quantum efficiency and field dependence are uniquely determined on an absolute scale, as is the temperature dependence at a fixed field. Such data are shown in Figure 3(b) for the sample prepared in He and are consistent with $r_{0} \sim 40 \AA$ in agreement with the fit in Figure 3(a). This particularly important piece of evidence will be discussed again when non-geminate recombination is considered. Very recently these measurements have also been carried out for the first time on $\mathrm{a}-\mathrm{As}_{2} \mathrm{Se}_{3}$. In this case an $\mathrm{r}_{\mathrm{o}} \sim 80 \AA$ 


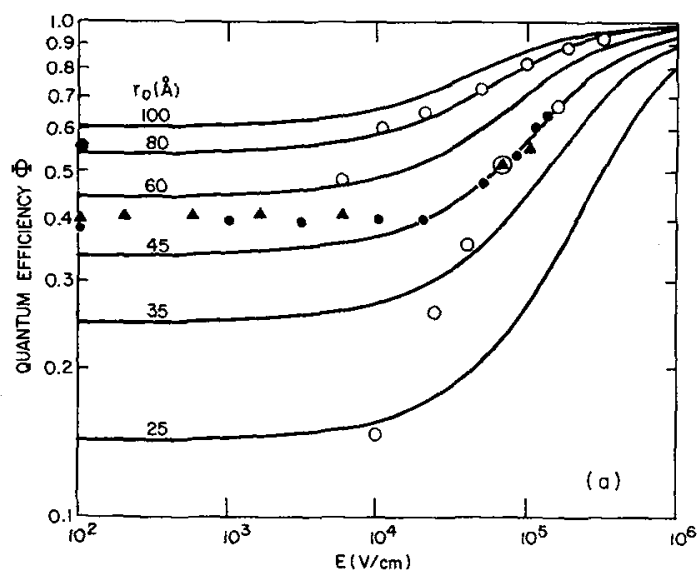

Fig. 3(a). Quantum efficiency of holes as a function of electric field for a-Si:H. The open circles refer to xerographic discharge measurements and the closed symbols to delayed-collection field experiments. The solid lines are the theoretical Onsager dissociation efficiencies for $\Phi_{O}=1, \varepsilon=11.5$ and for the different values of $\mathrm{T}_{O}$ indicated.

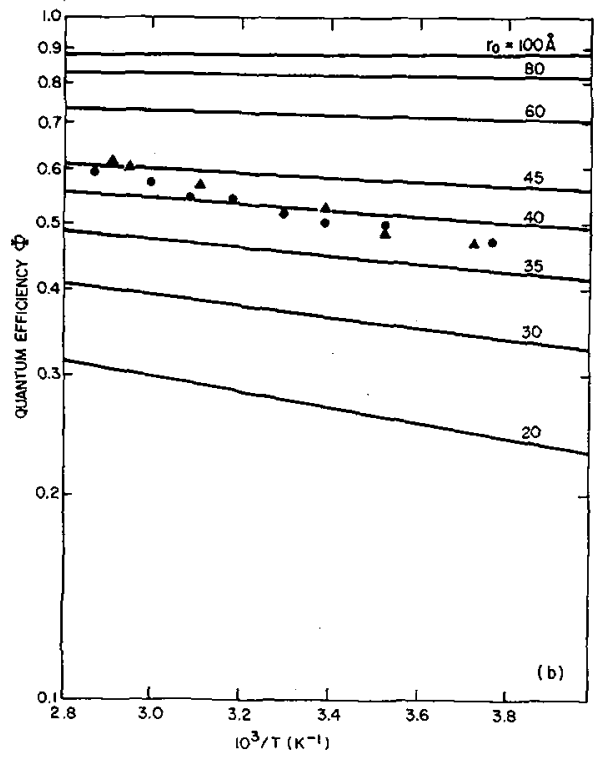

Fig. 3(b). Quantum efficiency versus reciprocal temperature. Solid lines are predictions of the Onsager theory (Ref. 17).

is found and like a-Si:H, but in contrast to a-Se, $r_{0}$ must be weakly wavelength dependent because a- $\mathrm{As}_{2} \mathrm{Se}_{3}$ has panchromatic photoresponse. With $\mathrm{r}_{0}$ determined $\Phi_{0}=1$ and with a dielectric constant of 9.0 the predicted temperature dependence of the Onsager theory is very weak which is confirmed experimentally.

\section{Non-Geminate Recombination}

As alluded to earlier the delayed-collection field technique allows a direct study of nongeminate recombination within the photogeneration region [18]. This is done by creating the carriers with a 10 nanosecond light flash in zero field. A high collection field which can be independently demonstrated to sweep out all remaining carriers is then applied after varying delays. In this way the carriers photogenerated (i.e. evade geminate recombination) in zero field with an efficiency of $\Phi(0)$ are allowed to co-exist and thus have the opportunity to trap and/or recombine as a function of the delay time $\Delta t$. The results of these experiments in an $a-S i: H$ sample at different temperatures are shown in Figure 4.

Since the carrier being studied are holes which exhibit dispersive transport [19] particular care was taken to ensure that a definable transit still occurred after the longest delay. In addition integration for as long as 100 transits was made so that essentially all transiting carriers are measured. In this way the effects of dispersive transport on this measurement are effectively excluded. The data shown in Figure 4(a) were analylzed using an analysis based on carriers diffusing in a semi-infinite slab with a diffusion constant $D$, a surface recombination velocity $v$ and a bulk carrier free lifetime of $\tau$. This analysis gives a fit indicated by the solid lines in figure 4(a). From this analysis it is possible to estimate the diffusion constant and hence the hole mobility (from the Einstein relation) and surface recombination velocity $\mathrm{v}$ as a function of temperature and this is shown in Figure (4b). The temperature dependence of both $\mu$ and $v$ 


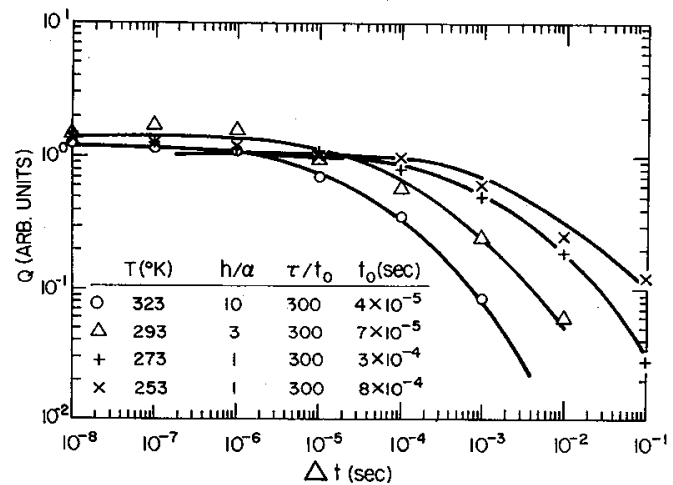

(a)

Fig. 4(a) Experimental results of collected charge $Q$ vs. delay time $\Delta t$ in $\mathrm{a}-$ $\mathrm{Si}: \mathrm{H}$. The solid curves are theoretical results obtained with the parameter values shown assuming diffusion of carriers in a semi-infinite slab (Ref. 18).

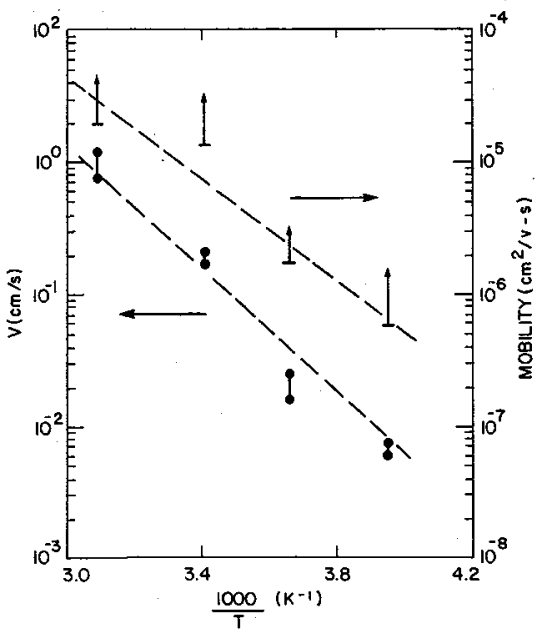

(b)

Fig. 4(b). Temperature dependence of the surface-recombination velocity and carrier mobility in a-Si:H deduced from delayed - collection field experiments (Ref. 18).

are shown as a function of temperature. The temperature dependence of both $\mu$ and $\mathrm{v}$ can be approximately described by an activation energy $\sim 0.4 \mathrm{eV}$. A similar activation energy for the hole drift mobility has been reported by independent time-of-flight measurements. This similarity in activation energy between recombination parameters and the hole drift mobility suggests that the non-geminate recombination in $\mathrm{a}-\mathrm{Si}: \mathrm{H}$ is a mobility or diffusion-controlled process such as Langevin recombination. The very small surface recombination velocity $1 \mathrm{~cm} / \mathrm{sec}$ at room temperature compared to the corresponding values in Si crystals of $10^{2} \mathrm{~cm} / \mathrm{sec}$ for a high quality surface and $10^{5} \mathrm{~cm} / \mathrm{sec}$ for a sand blasted surface is consistent with the surface recombination velocity being controlled by diffusion to that surface. With this perspective the essentially temperature independent quantum efficiency measured for delay times $\sim 50$ nanoseconds shown in figure 3(b) implies that the carrier loss measured under these conditions does not arise from non-geminate recombination in the photogeneration region but rather from geminate recombination loss. Similar studies of the non-geminate recombination as a function of temperature have recently been made for $\mathrm{a}-\mathrm{As}_{2} \mathrm{Se}_{3}[20]$ and at room temperature have been reported by Enck for a-Se [21]. A summary of values for the bulk lifetime and surface recombination velocity at room temperature for $\mathrm{a}-\mathrm{Se}, \mathrm{a}-\mathrm{Si}: \mathrm{H}$ and $\mathrm{a}-\mathrm{As}_{2} \mathrm{Se}_{3}$ are summarized in Table 1.

Table 1

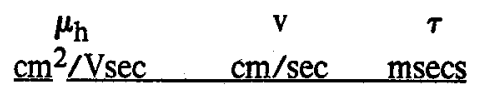

$\begin{array}{llcc}\text { a-Se } & 0.1 & 150 & - \\ \text { a-Si:H } & 10^{-4} & 10^{-1} & 10 \\ \text { a- } \mathrm{As}_{2} \mathrm{Se}_{3} & 10^{-7} & 10^{-3} & 1000\end{array}$


Figure 5 shows a composite plot for the non-geminate recombination data at room temperature for these three amorphous solids. It is striking that this illustrates the fact, deducible also from Table 1 , that the recombination rate appears to correlate with the value of the hole drift mobility for the three materials. In terms of a Langevin process where the rate is proportional to $\mu_{e}+\mu_{h}$ this is immediately understandable for a-Se and $\mathrm{a}-\mathrm{As}_{2} \mathrm{Se}_{3}$ where in each case the hole drift mobility is larger by orders of magnitude than the electron drift mobility. The

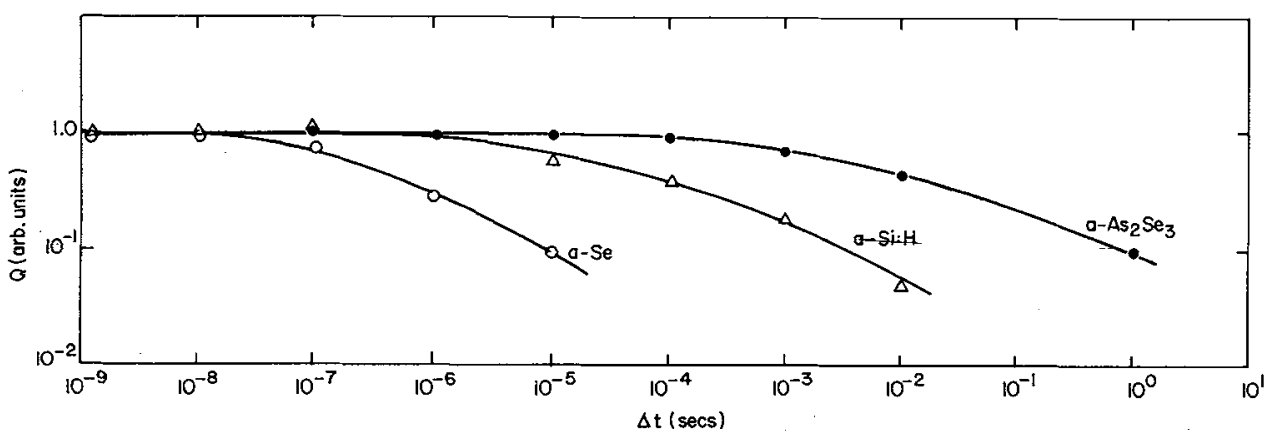

Fig. 5. Collected charge $Q$ as a function of delay time $\Delta t$ for a-Se (Ref. 21), a-Si:H (ref. 18) and a-Ass $\mathrm{Se}_{3}$ (Ref. 20).

converse is true, however, for a-Si:H. This can be understood however if either the higher mobility electrons diffuse independently to the surface or rapidly deep trap before undergoing recombination. Consistent with this, is the fact that time-of-flight studies show the electron deep trapping lifetime to be quite short $<300$ nanoseconds. These non-geminate recombination lifetime studies indicate that recombiration lifetimes can be as long as 10 mseconds for a-Si:H and $1 \mathrm{sec}$ for $\mathrm{a}-\mathrm{As}_{2} \mathrm{Se}_{3}$. Samples for a-Si:H have been studied which exhibit significantly shorter lifetimes than $10 \mathrm{mseconds}$ indicating a sensitivity to the quality and defect densities in the samples. Recombination lifetimes for holes $\sim 10$ mseconds, however, yield hole diffusion lengths $\sqrt{ } \mathrm{D}_{\tau}>0.6 \mu \mathrm{m}$. These lifetimes and diffusion lengths resulting from the delayedcollection field experiments are, because of the limitations of the experiment, limited to relatively low carrier densities $\sim 10^{15}$ carriers/cc.

\section{Discussion}

The combined use of xerographic discharge and delayed-collection field experiments have been applied to a-Se, a-Si:H and $\mathrm{a}-\mathrm{As}_{2} \mathrm{Se}_{3}$. In all cases the data are consistent with a geminate-recombination-controlled photogeneration process. Prior to this work a number of somewhat indirect studies had also led to this suggestion. As discussed by Williams and Crandall [22] the occurrence of such a process in amorphous solids is likely to be important because of the low microscopic mobilities. With reasonable expectations of a microscopic mobility $10 \mathrm{~cm}^{2} / \mathrm{Vsec}$ and a thermalization time $\sim 10^{-12} \sec$ then $\mathrm{r}_{\mathrm{o}} \sim \sqrt{ } \mathrm{D} \tau \sim 50 \AA$. Despite this a-Si:H and $\mathrm{a}-\mathrm{As}_{2} \mathrm{Se}_{3}$ have panchromatic photosensitivity which precludes. the strong wavelength dependence of $r_{0}$ found in a-Se.

The consequences of a geminate-controlled photogeneration process revolve around the predicted field dependent photogeneration. Such an effect is of minimal importance in

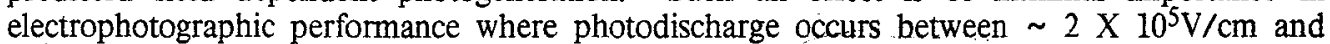
$10^{4} \mathrm{~V} / \mathrm{cm}$, the latter resulting in a very little residual or undischarged voltage. As a result the average photogeneration efficiency throughout the discharge is $\sim 0.8$. A geminate process 
would, however, have more effect on solar cell efficiencies. In the photovoltaic cells the potential barriers result in spatial field distributions. Since the photogeneration efficiency is field dependent it therefore becomes spatially dependent on the light absorption profile. Such behavior can be modelled [23]. Table 2 summarizes the results of these calculations for the short-circuit current $\mathbf{J}_{s c}$, open-circuit-voltage $V_{o c}$ and the maximum output power $P_{\max }$.

Table II

\begin{tabular}{lcccc}
\multicolumn{1}{c}{$\eta$} & $\begin{array}{c}\mathrm{J}_{\mathrm{sc}} \\
\mathrm{mA} / \mathrm{cm}^{2}\end{array}$ & $\begin{array}{c}\mathrm{V}_{\mathrm{oc}} \\
\text { volt }\end{array}$ & $\begin{array}{c}\mathrm{P}_{\max } \\
\left(\mathrm{mW} / \mathrm{cm}^{2}\right)\end{array}$ & $\mathrm{FF}$ \\
\hline $\begin{array}{l}\mathrm{f}_{\mathrm{O}}=\mathrm{f}_{\mathrm{t}}=1 \\
\mathrm{n}=1\end{array}$ & 32.6 & 0.588 & 14.9 & 0.766 \\
$\eta(\mathrm{E}, 80)$ & 20.7 & 0.573 & 8.61 & 0.726 \\
$\eta(\mathrm{E}, 60)$ & 17.2 & 0.568 & 6.97 & 0.713 \\
$\eta(\mathrm{E}, 50)$ & 14.8 & 0.564 & 5.86 & 0.703 \\
$\eta(\mathrm{E}, 40)$ & 11.7 & 0.555 & 4.50 & 0.689 \\
& & & & \\
\hline $\mathrm{f}_{\mathrm{o}}=0, \mathrm{f}_{\mathrm{t}}=1$ & & & & \\
$\eta=1$ & 17.3 & 0.570 & 7.39 & 0.749 \\
$\eta(\mathrm{E}, 80)$ & 11.4 & 0.553 & 4.31 & 0.685 \\
$\eta(\mathrm{E}, 60)$ & 9.49 & 0.547 & 3.54 & 0.682 \\
$\eta(\mathrm{E}, 50)$ & 8.17 & 0.543 & 3.01 & 0.679 \\
$\eta(\mathrm{E}, 40)$ & 6.51 & 0.537 & 2.36 & 0.676
\end{tabular}

As can be seen $\mathrm{J}_{\mathrm{sc}}$ and $\mathrm{P}_{\max }$ are significantly reduced by a geminate process while $\mathrm{V}_{\mathrm{oc}}$ and the fill factor FF are only slightly affected. Since $f_{o}$ and $f_{t}$ are the fractions of light internally reflected within the cell at the front and rear boundaries respectively, the condition $f_{0}=f_{t}=1$ is where all incident photons are absorbed. The case where $f_{0}=0$ and $f_{t}=1$, i.e. the light makes two passes in the undoped a-Si:H layer is more realistic. In the latter case the maximum short circuit current $\sim 17 \mathrm{ma} / \mathrm{cm}^{2}$ while with a geminate process and $\mathrm{r}_{0} \sim 80-100 \AA$, $\mathrm{J}_{\mathrm{sc}}$ is reduced to $\sim 11-12 \mathrm{ma} / \mathrm{cm}^{2}$ [24]. These are about the maximum short-circuit currents measured in reported solar cells and would mean that other recombination losses are negligible. This seems consistent with the inapplicability of a minority carrier lifetime concept in what is a photoconducting insulator. This implies that any non-geminate recombination of the ShockleyRead type will be controlled by the longer lifetime. The non-geminate recombination studies reported in this paper suggest that rather long, $\sim 10 \mathrm{msecs}$, lifetimes for holes in a-Si:H can exist which suggest diffusion lengths $>0.5 \mu \mathrm{m}$. This conclusion is consistent with recent measurements due to Dresner [25]. The latter results are of specific interest because these measurements were made at 1 sun. The values at 1 sun and the low intensity of the delayed collection field are consistent with the relative insignificance of non-geminate recombination loss and the lack of non-linearity in light intensity of the short-circuit current. Despite this apparent consistency between the relative roles of geminate and non-geminate recombination losses and a-Si:H photovoltaic performance, it is difficult to reconcile the reported observation of collection 
efficiencies $\sim 0.9$ at $5000 \AA$ [26] if this quantity is measured to better than $\pm 10 \%$. The only way such a collection efficiency could occur together with a geminate photogeneration process are, (a) $r_{o}$ in some samples is large (calculations indicate $r_{o}$ would have to be $\sim 500-600 \AA$ which in turn leads to microscopic mobilities $\sim 1000 \mathrm{~cm}^{2} / \mathrm{Vsec}$ ), (b) unusually high effective dielectric constants within the barrier region due to the space charge (calculations indicate $\varepsilon_{\text {eff }} \sim 100$ is required) and (c) at solar intensities the photoexcited carrier density is such that self-screening precludes the existence of a geminate pair description (this process however would lead to deviations from linearity of the short circuit current on light intensity at low intensities).

Geminate recombination and its complementary non-geminate process, Langevin recombination, should be expected to operate in $\mathrm{a}-\mathrm{Si}: \mathrm{H}$ as well as a-Se and $\mathrm{a}-\mathrm{As}_{2} \mathrm{Se}_{3}$. There is strong experimental evidence that such a process occurs in a-Se and recent measurements give comparable evidence for a-Si:H and $\mathrm{a}-\mathrm{As}_{2} \mathrm{Se}_{3}$. In one important respect, however, both these materials differ from a-Se in that they are panchromatic in photosensitivity which means that the thermalization length is at most weakly wavelength dependent. The existence of a geminate process in a-Si:H could be significant for its functioning as a photovoltaic solar cell. While this has been analytically modelled and can account for the observed short-circuit currents it is inconsistent with the observation of collection efficiencies of 0.9 at $5000 \AA$. A number of possible ways of reconciling these conflicting observations have been mentioned but it is not yet possible to account for the discrepancy.

Acknowiedgements: The author would like to acknowledge the contributions to the work done at Xerox which is discussed in this paper by I. Chen, S. Grammatica, J. Knights, R. Lujan, M. Morgan and A. Troup. Discussions with D. Pai were most helpful.

\section{References}

[1] CARLSON, D.E. and WRONSKI, C.R., Appl. Phys. Letters 28 (1976) 671.

[2] SPEAR, W.E., Advances in Physics, 26 (1977) 811.

[3] GIBSON. R.A., SPEAR., W.E., LeCOMBER, P.G. and SNELL; A.J., J. Non-Crystl. Solids 35-36 (1980) 725 .

[4] LeCOMBER, P.G., SPEAR, W.E. and GAITH, A., Electronic Letters 15 (1979) 179.

[5] SHIMIZU, I., KOMATSU, T., SAITO, E. and INOUE, E., J. Non-Crystl. Solids 35-36 (1980) 773 .

[6] MORT, J, GRAMMATICA, S., KNIGHTS, J.C. and LUJAN, R., Photogr. Sci. Eng. 24 (1980) 241.

[7] FRITZSCHE, H., Solar Energy Materials 3 (1980) 447.

[8] HUGHES, R.C., J. Chem. Phys. 58 (1973) 2212.

[9] SPEAR, W.E., Amorphous and Liquid Semiconductors, edited by Stuke, J. and Brenig W., Taylor and Francis, London, 1974.

[10] TIEDJE, T., CEBULKA, J.M., MOREL, D.L. and ABELES, B., Phys. Rev. Letters (to be published). 
[11] ONSAGER, L., Phys. Rev., 54 (1938) 544.

[12] ROSE, A., Concepts in Photcconductivy and Allied Problems, Interscience Publishers, New York 19673).

[13] DOLEZALEK, F.K. and SPEAR, W.E., J. Phys. Chem Solids, 36 (1975) 819.

[14] LANGEVIN, P., Ann. Chem. Phys. 28 (1903) 289, 443.

[15] PAI, D. and ENCK, R., Phys. Rev., B11 (1975) 5163.

[16] KNIGHTS, J.C. and DAVIS, E.A., J. Phys. Chem. Solids 35 (1974) 543.

[i7] MORT. J., TROUP. A., MORGAN, M., GRAMMATICA, S., KNIGHTS, J.C. and LUJAN, R., Appl. Phys. Letters, 38 (1981) 277.

[18] MORT. J., CHEN, I., TROUP. A., MORGAN, M., KNIGHTS, J.C. and LUJAN, R., Phys. Rev. Letters 45 (1980) 1348.

[19] MORT. J., GRAMMATICA, S., KNIGHTS, J.C. and LUJAN, R., Solar Cells, 2 (1980) 451 .

[20] MORT. J., CHEN, I., MORGAN, GRAMMATICA, S., Solid State Comm (to be published).

[21] ENCK, R.C. and PFISTER, G., in Photoconductivity and Related Phenomena, Chapter 5, edited by Mort. J. and Pai, D., Elsevier Scientific Publishing, Amsterdam (1975).

[22] WILLIAMS R. and CRANDALL, R.S., RCA Review, 40 (1979) 371.

[23] CHEN, I. and MORT. J,, Appl. Phys. Letters 37 (1980) 952.

[24] CARLSON, D.E., Solar Energy Materials 3 (1980) 503.

[25] DRESNER, J. SZOSTAK, D.J. and GOLDSTEIN, B., Appl. Phys. Letters (to be published.

[26] CARLSON, D.E., (private communication). 